\title{
Networking Mass Spectrometer Data Systems for Improved Productivity and Electronic Archiving of Data
}

\author{
Mark J. Hayward, Paul V. Robandt, Jon T. Meek, and Michael L. Thomson \\ American Cyanamid Company, Agricultural Research Division, Analytical, Physical and Biochemical Research \\ Section, Princeton, New Jersey, USA
}

\begin{abstract}
Several Finnigan-MAT mass spectrometer data systems were networked together to achieve the following two primary objectives: (1) to allow access to mass spectrometry data and data processing functions from remote locations without affecting simultaneous data acquisition at the instruments, and (2) to electronically archive mass spectrometry data at a central location on a high-capacity, fast-access device that allows rapid retrieval of archived data for all data processing operations at all locations. UNIX workstations, IBM PC/AT-compatible computers, and Data General Nova minicomputers were connected via Ethernet interfaces to allow rapid data transfer among all systems as well as X-Windows access to UNIX-based systems. Bridging techniques were used to isolate possible high-traffic areas of the network and to enable security measures for adequate protection of files. Additionally, serial connections were made through a Northern Telecom phone system to provide remote terminal access to the Data General Nova-based systems. Use of these connectivity techniques significantly improved productivity by allowing retrieval, processing, and printing of data from remote locations, such as office areas, without affecting data acquisition, processing, and printing performed simultaneously at the instruments. For archival purposes, data files are electronically stored on high-capacity magneto-optical disks for rapid retrieval. A highcapacity fixed disk is also available for centralized temporary data file storage. A Digital Equipment Corporation DECstation 2100 UNIX workstation was used as the file server for centralized data storage while being simultaneously utilized as the data system computer for one of the mass spectrometers. Utilization of this UNIX-based file server system in conjunction with Ethernet connectivity techniques provides a centralized, rapid-access, high-capacity, cost- and space-efficient method for electronic archival of mass spectrometry raw data recorded at all of the instruments. (J Am Soc Mass Spectrom 1993, 4, 742-750)
\end{abstract}

$\mathrm{T}$ The connection of computers via networking interfaces is rapidly expanding and improving the ways in which computers are used. Computer networks are becoming commonplace in office operations and data processing environments. The driving force behind these connectivity methods has been the need for rapid and cost-effective access to information at many locations. Improved access to vital information often benefits strategically competitive organizations by increasing job efficiency and lowering operating costs.

Minimizing costs and maximizing efficiency are becoming increasingly important to businesses that aim to produce technologically based, distinctly competent products. Therefore, similar needs for rapid access to information also exist for organizations that depend on technical or scientific data. Improved inter-

Address reprint requests to Mark J. Hayward, American Cyanamid Company, Agricultural Research Division, Analytical, Physical and Biochemical Research Section, P.O. B ox 400, Princeton, NJ 08543-0400. nal access to scientific and engineering data can help promote more efficient and lower cost research and development by making some of these operations easier and less time-consuming. In addition, the availability of the archived electronic raw data set can facilitate reinterpretation of existing research data, which can, in some cases, be used to reach new conclusions without the need for costly supplementary studies. Furthermore, many technology-based businesses face increasingly stringent regulatory demands that require complete records of product registration-related studies to be archived and readily available for government inspection. As a result, the long-term availability of raw data in their electronic form, in addition to the traditional hard copy, is rapidly becoming critical to supporting a company's position. The application of computer networking techniques to scientific instrument data systems can provide the required access and archival capabilities to promote high efficiency and long-term availability of raw data to support product registration positions. Aware of the potential benefits, 
the Analytical, Physical and Biochemical Research Section at the Agricultural Research Division of Anterican Cyanamid Company initiated the systematic development of a laboratory information management system (LIMS) that addresses these access and availability needs for all of our analytical data. The purpose of this report is to describe the details of one of the first steps that we took in that initiative, which involves the implementation of techniques intended to achieve these benefits for our mass spectrometer data systems.

The effective use of computer networking with scientific instrumentation is in its infancy. Integrating many diverse data systems is an important challenge [1] facing both end users and instrument manufacturers. The manufacturers are striving to provide the appropriate hardware for developing connectivity techniques [1-3]. This effort is evident in the recent appearance of many new LIMS products. At the same time, some progress is also being made in the standardization of data formats [4-6] and the evolution of software for information management. These developments are beginning to spawn end-user interest in file transfer [7] and archival techniques for mass spectrometry data [8]; however, computer networking techniques facilitate new capabilities that extend well beyond data file transfer and storage. This report describes the specifics of how we applied networking techniques to maximize the capabilities of our existing mass spectrometer data systems.

\section{Instrumentation}

The following mass spectrometer and data system combinations were networked together: (1) a Finnigan-

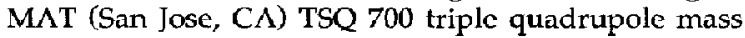
spectrometer with an Interactive Chemical Information System (ICIS) data system based on a Digital Equipment Corp. (DEC, Maynard, MA) DECstation 2100 workstation; (2) a Finnigan-MAT TSQ 46 triple quadrupole mass spectrometer with an INCOS data system based on a Data General (DGC, Southboro, MA) Nova $4 \mathrm{X}$ minicomputer; (3) a Finnigan-MAT TSQ 45 triple quadrupole mass spectrometer with an INCOS data system based on a Data General Nova $4 \mathrm{X}$ minicomputer; and (4) a Finnigan-MAT Magnum ion trap detector with a data system based on a Gateway-2000 486/33C IBM PC/AT-compatible computer (Gateway 2000, North Sioux City, SD).

The DECstation 2100 workstation is equipped with 24 Mbytes of memory, an Ethernet interface, a 17-in. color monitor (DEC VR290-DA), two 330-Mbyte fixed disk drives (DEC rz55), a 1.2-Gbyte fixed disk drive (Micropolis 1598, which is equivalent to a DEC rz57), a 650-Mbyte magneto-optical disk drive (Sony SMOE501), a 50-Mbyte tape drive (DEC tk50), and a PostScript laser printer (DEC $\ln 03$ ). The DECstation 2100 utilizes the Ultrix 4.1 operating system (DEC's version of UNIIX) and the version 7.0 ICIS software.
Each of the two Data General Nova 4X minicomputers is equipped with $256 \mathrm{Kbytes}$ of memory, a second terminal option with a Nurthern Telecom (NT, Santa Clara, CA) QMT8 Asynchronous Data Module (ADM) connected, an Ethernet interface board (Claflin \& Clayton, Northboro, MA), a 96-Mbyte fixed disk drive with a 16-Mbyte removable cartridge (Control Data CMD 96), a high-speed dot matrix line printer (Printronix P300, Printronix, Irvine, CA), and a CompuAdd (CompuAdd, Austin, TX) 212 IBM PC/ AT-compatible computer used as a Tektronix 4010 terminal. The EM4010 (Diversified Systems, Boulder, $\mathrm{CO}$ ) program is used to emulate a Tektronix 4010 terminal with the CompuAdd 212 computers. The Data General Nova $4 \mathrm{X}$ minicomputers utilize the version 6.1 SuperINCOS and the version 2 (beta) Claflin \& Clayton Trivial File Transfer Protocol (TFTP) software.

The Gateway-2000 $486 / 33 \mathrm{C}$ computer is equipped with 8 Mbytes of memory, a 120-Mbyte fixed disk drive, 3.5- and 5.25-in. floppy disk drives, an ATI Ultra 8514 / A video card with 1 Mbyte of memory, a Crystal Scan 1024 NI 14-in. super-VGA monitor, an 8-bit 3COM 3C503 EtherLink II interface card, and a QMS PS815 printer. The Gateway-2000 486/33C utilizes the Microsoft (Microsoft, Seattle, WA) Disk Operating System (MS-DOS) version 5.0, SUN Microsystems (SUN, Mountview, CA) PC-NFS version 4.0, Hummingbird Communications (HCL, Markhan, Ontario, Canada) $X$-Window server HCL eXceed HiRes version 3.1, and version 2.1 Magnum Ion Trap system software.

All network connections are made with the appropriate cable for Ethemet IEEE 802.3 standard 10BASE5 or 10BASE2 physical layer alternatives. A Retix (Retix, Santa Monica, CA) $4660 / \mathrm{S}$ network bridge is used to limit unnecessary network traffic and to enable access security to mass spectrometry data files.

Offices are equipped with SUN SPARCstation IPC workstations with 24 or 36 Mbytes of memory, a 16-in. color monitor, and a laser printer with both HP PCL and PostScript capability. The SUN IPC workstations utilize the version 4.1 SUN-OS operating system and the OpenWindows 3 windowing system. The offices are also equipped with $80386 / 25-\mathrm{MHz}$ or $80486 /$ $33-\mathrm{MHz}$ IBM PC/AT-compatible computers with 8 Mbytes of memory; an STB (STB Systems, Richardson, TX) PowerGraph video card that is set to the 16-bit, no interrupts, noninterlaced mode with 1 Mbyte of on-board memory; a 15-in. NEC (NEC, Tokyo, Japan) MultiSync 3FGx monitor; and an 8-bit 3COM 3C503 EtherLink II interface card. The personal computers (PCs) utilize MS-DOS version 5.0, SUN Microsystems PC-NFS version 4.0 , and Hummingbird Communications $X$-windows server HCL eXceed Plus version 3.1. All department PCs and some workstations are equipped with Northern Telecom QMT9 Asynchronous Interface Modem (AIM) to permit data communication with the SuperINCOS data systems via the Northern Telecom Meridian SL-1/XT phone system. 


\section{Results}

\section{Using a UINIX Workstation-Based Data System as a File Server for Data Archival}

UNIX workstation computers often have advantages over other computers that have been used for instrument data systems. The benefits of using these workstations include the hardware's ability to rapidly execute program instructions and the multitasking, multiuser operating system that can simultaneously execute many programs. Such an environment allows the data system to be utilized by multiple users for data acquisition, data processing, and file and printer management.

The ICIS data system based on the DECstation 2100 UNIX workstation and used with the Finnigan-MAT TSQ 700 mass spectrometer has considerable capability as a network server in addition to its known ability for mass spectrometry applications. These capabilities can be used for the processing, management, and archival of data from other mass spectrometers, in addition to data from the TSQ 700. To utilize these resources, the mass spectrometer data systems for the Finnigan-MAT TSQ 700, TSQ 46, TSQ 45, and Magnum instruments were connected via Fthernet interfaces, as shown in Figure 1.

Using the DECstation 2100 for purposes other than a single-user mass spectrometer data system requires expansion and reorganization of the file system to improve flexibility. It is desirable to put the Ultrix operating system (DEC's version of UNIX) files on a physically separate fixed disk drive because the Finnigan-MAT installation procedure repartitions and reformats the device where the files are installed. Installation of the Ultrix files on a device that is sepa- rate from all other files allows the upgrading of Ultrix features or versions without affecting other files or applications. Furthermore, the Ultrix software supplied with the DECstation is very large and can easily fill the standard 330-Mbyte fixed drive (rz55) if anything significantly more than the minimal installation is chosen. Therefore, two 330-Mbyte (rz55) fixed drives were used for the normal operation of the DECstation 2100 data system. One of the $\mathrm{rz} 55$ drives contains the " $/$ " and the "/usr" partitions and is used only for the files supplied by DEC with the Ultrix operating system. The second rz55 drive is mounted as "/usr/users" and contains all user $\log$-in directories and all additional applications, such as the ICIS data system software. All applications directories located below "/usr/users" have symbolic links connecting them to "/usr," thereby preserving the original directory philosophy as supplied by Finnigan-MAT.

Use of the DECstation 2100 as a file server places increased demand on the file system for storage space. Therefore, an additional fixed drive, 1.2 Gbyte (rz57). was added to the DECstation 2100 for use as temporary storage for data files from all of the mass spectrometer data systems on the network and is mounted as "/usr/users/netdrive." To have relatively highspeed, high-capacity media for permanent archival of mass spectrometry data files, a 650-Mbyte optical disk drive (Sony SMO-E501) was installed and is mounted as "/usr/users/optical." Organization and expansion of the file system as described permits the use of the DECstation 2100 as a flexible multiuser system for data acquisition, data processing, file serving, print scrving, and data archival.

The Data General Nova $4 \mathrm{X}$ data systems for the Finnigan-MAT TSQ 45 and TSQ 46 mass spectrometers were interfaced to the Ethernet network using interface

\section{Ethernet $1 O B A S E 2$}

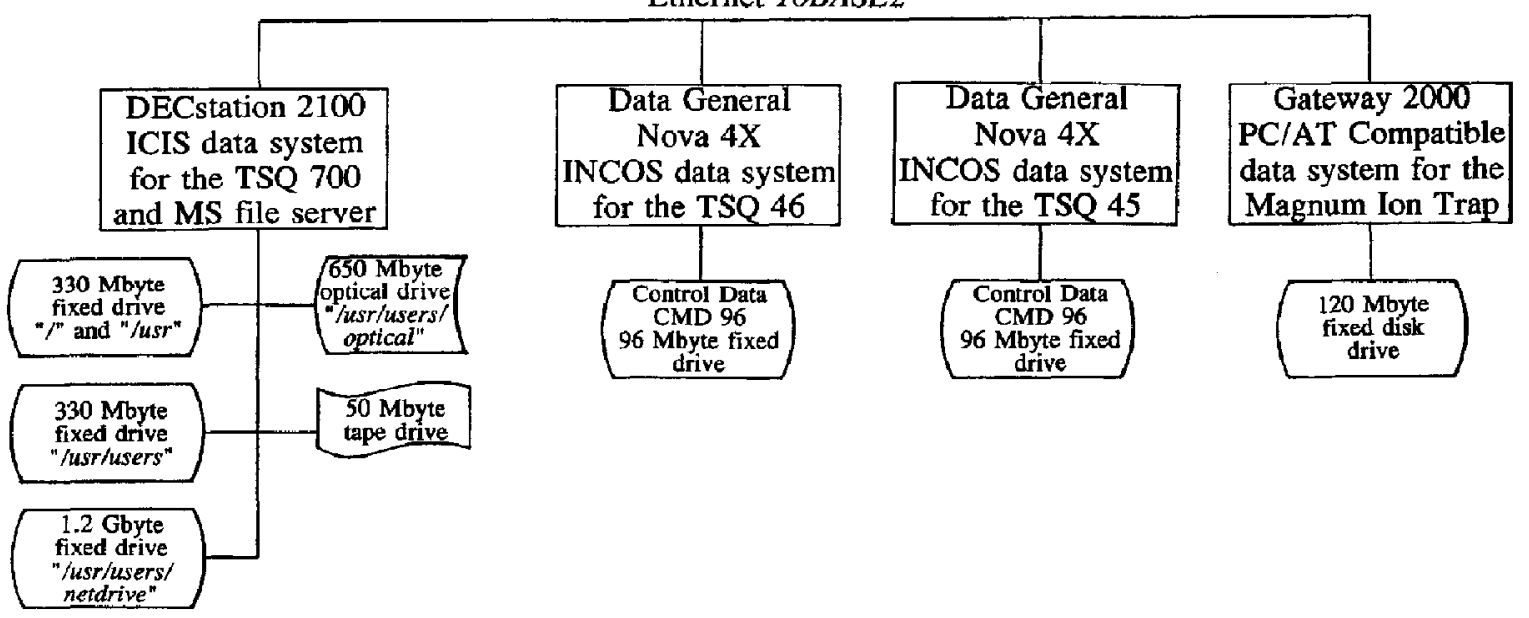

Figure 1. Schematic illustrating the Ethernet connection of four mass spectrometer data systems, each shown with its respective file system. The DECstation 2100 workstation is utilized as both a mass spectrometer data system and file server for the storage of data files from all of the mass spectrometers. 
boards and software from Claflin \& Clayton sold by Finnigan-MAT. Files are transferred by a simple SuperINCOS procedure (script) that uses the TFTP. SuperINCOS time versus intensity data files are converted to a mass versus intensity format using the "ENHA" command prior to TFTP transfer to allow the

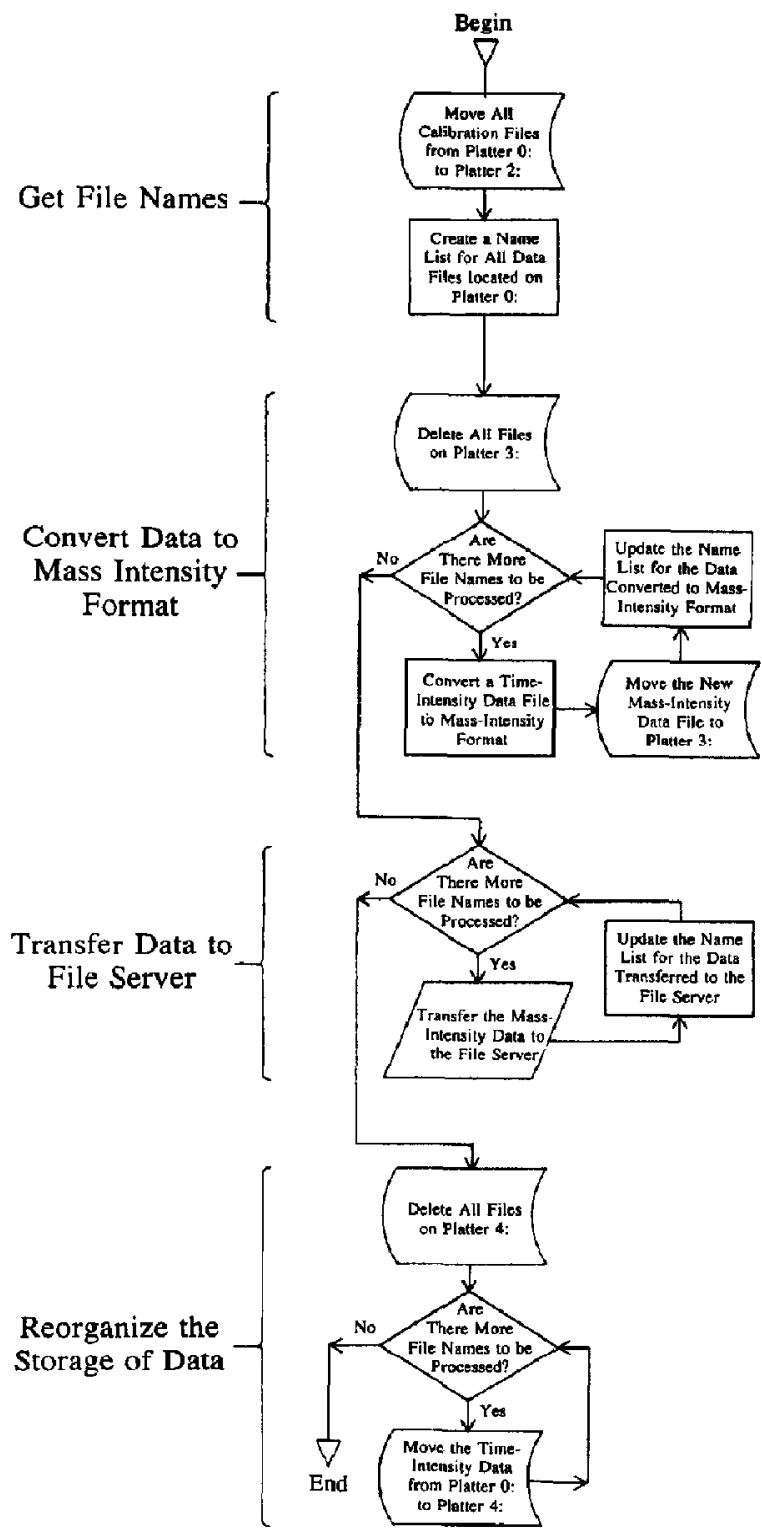

Figure 2. Flow diagram depicting the execution of the SuperINCOS procedure that is used to transfer data from the Data General Nova 4X minicomputers to the DECstation 2100 workstation. This procedure is also used to administrate and organize the storage of data on the CMD drives used with the Data General computers. Note that subprocedures (indicated by braces) were selectively utilized in the procedure's creation so that each subprocedure can be executed separately for archiving older data, originally stored on CMD disk packs, onto modern media. data to be processed by the more modern FinniganMAT data systems. A flow diagram of the SuperINCOS procedure used for routine archival of INCOS data at the DECstation 2100 is shown in Figure 2. Successful use of this procedure requires adherence to a naming scheme for data files. The "FILE(> filename ( modifier; E)" command is used to create a name list of file names that fit our naming scheme. Also note that data files are buffered from platter 0: to platter 4: of the Control Data CMD96 drive to provide the maximum duration of availability of data on each of the data systems. The procedure shown in Figure 2 is typically executed on a biweekly to monthly basis (based on instrument use) and provides complete administration of the INCOS file system as well as electronic archival of all INCOS data on modern media. Each of the component functions of this procedure is contained in its own subprocedure that can be executed separately as needed. Separate execution of each subprocedure can be especially useful when archiving older data that do not adhere to a naming scheme for data files or for data that have become separated from their calibration table. The use of these subprocedures, along with some selected editing of the name lists created when they are executed, has allowed archival of all cata originally saved on CMD96 disk packs since 1982 (when the first INCOS system was purchased) to be made immediately available at the DECstation 2100 and archived on optical disk.

Use of the standard TFTP services that are supplied with the DECstation 2100 are not adequate for routine transfer of files because files may only be written if the file names preexist and the files are publicly writable. Therefore, the TFTP server by Stevens ${ }^{1}$ [9] was modified to allow files to be written to the DECstation 2100 without the restriction of having the files already existing in the target directory. To further facilitate the overall transfer process, the Stevens TFTP server was also modified so that uppercase file names are converted to lowercase, and the " $\$$ " character is stripped from the file name. The TFTP server mounts the file system at "/usr/users/netdrive" and permits unrestricted TFTP read and write file access below that point. File system protection is available by using the Retix $4660 / \mathrm{S}$ bridge in the security mode, thereby preventing access by all unapproved hosts. Note that TFTP transfers support only minimal capabilities for error checking or for resending data packets. Therefore, it is desirable that the DECstation be ready and waiting with sufficient central processing unit (CPU) and communication bandwidth to carry out the complete transfer at the time that it is requested. As a result, it is preferable that the ICIS instrument control program, which has the highest execution priority and is very demanding on CPU and communication time, be closed prior to TFTP transfers (i.e., the TSQ 700 is

\footnotetext{
${ }^{1}$ The modified version of Stevens' "tftp" was originally developed on a Sun SPARCstation and then was adapted to the DECstation.
} 
not acquiring data during TFTP transfers) to maintain the highest degree of reliability.

The IBM PC/AT-compatible data system for the Finnigan-MAT Magnum ion trap detector is interfaced to the Ethernet network using an 8-bit 3COM EtherLink II interface card and SUN PC-NFS sottware. With the Network File Server (NSF) daemon activated on the DECstation 2100, PC-NFS is used to mount "/usr/users/netdrive" on the DECstation 2100 as a logical MS-DOS drive at the PC-based Magnum data system. File transfer can be accomplished by simply copying files to the NFS drive or by using the NFS drive as the target drive for storing the data during acquisition.

\section{Incorporating Mass Spectrometer Data Systems into a Local Area Network}

The application of computer network techniques to mass spectrometer data systems at American Cyanamid Company started with stand-alone mass spectrometers, where the only network connection was the Fthernet line between the Finnigan-MAT TSQ 700 mass spectrometer and its DECstation 2100 data system. The initial goal of connecting the mass spectrometer data systems using Ethernet networking techniques was intended to provide a simple and cost-effective means of electronically storing the data recorded at each of the instruments. This interest eventually resulted in the Ethernet connection of the mass spectrometer data systems and other computer equipment, as shown in Figure 3. The link between the mass spectrometer data systems and the existing sitewide network was originally made to allow sharing of file storage capabilities, but, once coupled, it was quickly found to have many other benefits. Among the additional valuable uses that have resulted from this connection are the ability to process and print data at remote locations, such as in the office areas, to quickly transfer data in formats that allow their inclusion in reports and other documents, and to utilize these and all other capabilities of the DECstation 2100 data system computer from remote locations while simultaneously acquiring data at the instrument.

Connecting several computer systems together in an Ethernet network requires consideration of the level of traffic that may be present on the network. In some cases, the issue of security with regard to access to computer systems, data, and other information requires careful attention. Both of these considerations were addressed by placing a Retix $4660 / \mathrm{S}$ bridge between the mass spectrometer data systems and all other computers on the network. For the data systems used in this application, the only significantly heavy network traffic occurs between the Finnigan-MAT TSQ 700 and its DECstation 2100 data system. During data acquisition, the TSQ 700 can require $35 \%$ of the Ethemet bandwidth (on an intermittent basis) to send data packets to the DECstation 2100. Use of the bridge allows connection of this instrument to the network without subjecting the entire network to its high level of traffic during data acquisition. Security can be important when using transfer protocols, such as TFTP, that are public and not secured. Security considerations with TFTP involve both protection of files and maintenance of proper access. TFTP operations by other hardware on the network can impede TFTP file transfers. This can be a problem on a network where terminal servers are used because these servers are frequently set up to transfer diagnostic or boot files via TFTP. For the data systems used in this application, security is implemented by specifying exactly which hosts may communicate across the bridge.

\section{Remate Access to Data General Nova (INCOS)- Based Data Systems}

The Data General Nova $4 \mathrm{X}$ Minicomputer (INCOS)based data systems utilized with the Finnigan-MAT ISQ 45 and TSQ 46 mass spectrometers do not permit terminal acress via an Fithernet ronnertion. These INCOS data systems allow two Tektronix 4010 terminals to be connected through RS-232C serial ports. IBM PC/AT-compatible computers running the terminal emulation program EM4010 are used as the primary terminals (connected to port 0 ) for each instrument and are located at the instrument console in the laboratory. Remote access to the second terminal is available via the Northern Telecom phone system currently in use on-site. Connection of the INCOS data systems to remote terminals through the Northern Telecom phone system is illustrated in Figure 4. Each PC and workstation located in an office area is equipped with a Northern Telecom QMT8 AIM. An AIM is a phone system-specific modem with minimal features. Each INCOS data system is equipped with a Northern Telecom QMT9 Asynchronous Data Module (ADM). An ADM is a phone system-specific, full-featured modem with automatic call answering and hang-up capabilities. The use of these phone system devices allows remote users to access all data processing and printing features of either INCOS data system. Note that there can be only one remote user at a time for each INCOS data system. For this particular application, the demand is sufficiently moderate that the use of each INCOS data system by anly one remote user at a time is rarely a limitation. The terminal emulation program Kermit is used for all remote accesses to the INCOS data systems because it provides Tektronix 4010 emulation and because its batch commands facilitate fully automated connections. The use of these techniques permits access to the INCOS data systems from all office PCs or workstations without affecting simultaneous data processing or acquisition that may be occurring in the laboratory. Furthermore, access is very simple and requires only a single command for entry and a single keystroke for exiting. 


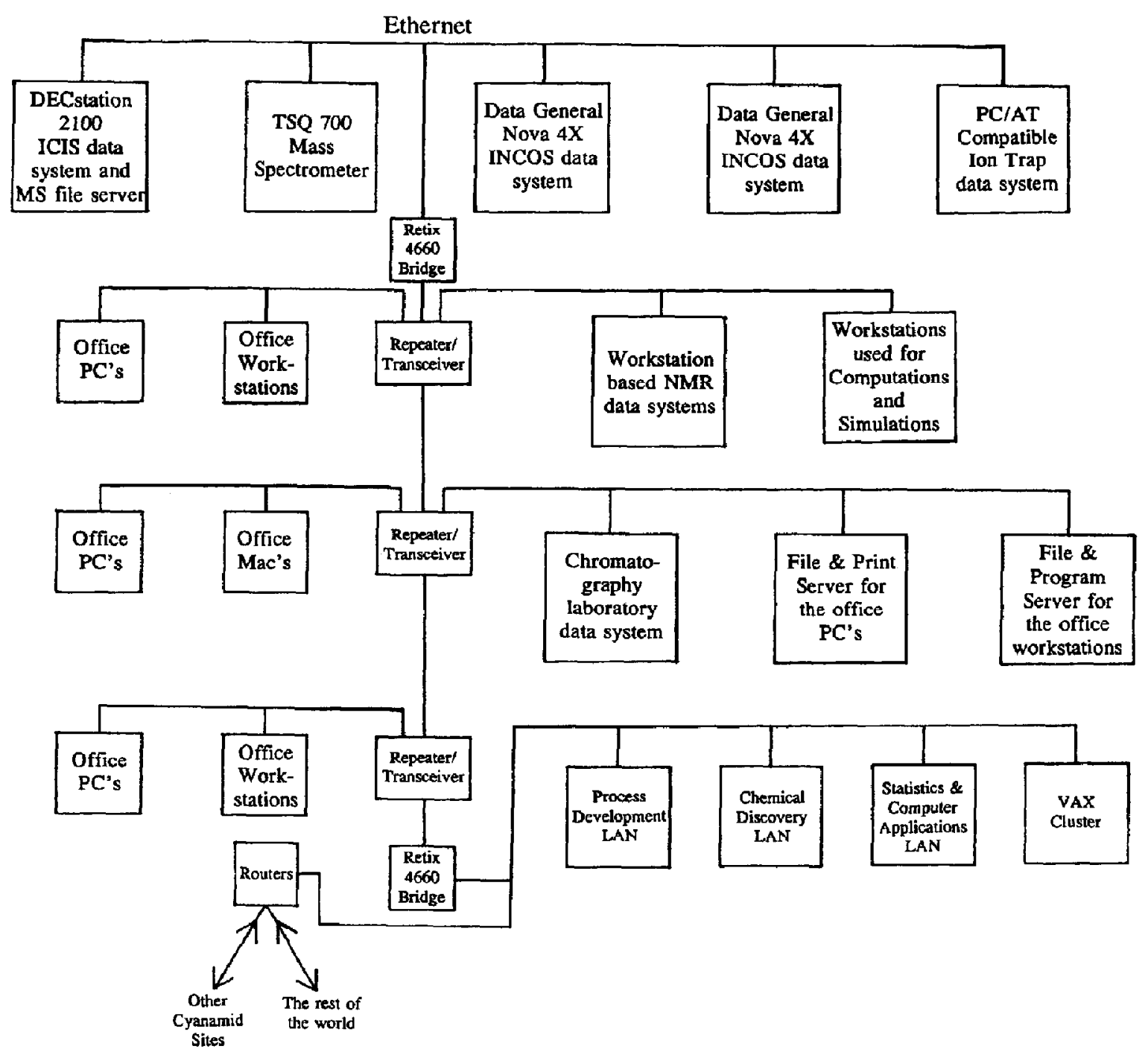

Figure 3. A mass spectrometrist's view of the Ethernet connection of the mass spectrometer data systems to the sitewide network at American Cyanamid's Agricultural Research Division. This link opens up many new possibilities in the transfer, display, processing, and printing of data. NMR, nuclear magnetic resonance; LAN, local area network; Mac, Macintosh.

\section{Remote Access to a UNIX Workstation-Based Data System}

Because many mass spectrometry analyses involve identifying unknowns in complex mixtures, it is essential to be able to reexamine the data, especially during discussions with the sample originator. The ability to quickly view, process, and print data in an office during the discussion of the results can provide immediate answers and facilitates the most productive and efficient use of time. The networking capabilities of the DECstation 2100 (ICIS) data system utilized with the Finnigan-MAT TSQ 700 mass spectrometer permits $X$-terminal access and printing from ICIS applications at remote locations, such as offices, via its Ethernet connection to the network. Remote access to the ICIS data system is made even more useful by virtue of the fact that the data files from all of the mass spectrometers are available at this data system.

As shown in Figure 5, a typical office is equipped with a PC, a UNIX workstation, and a laser printer. Both the PC (IBM PC/AT-compatible 80386/25 MHz or $80486 / 33 \mathrm{MHz}$ ) and the UNIX workstation (SUN SPARCstation IPC) are connected to the network, which includes the ICIS data system, through an Ethernet interface. The printer is connected to the UNIX workstation through a Centronics parallel port, with the UNIX workstation also functioning as a print server 


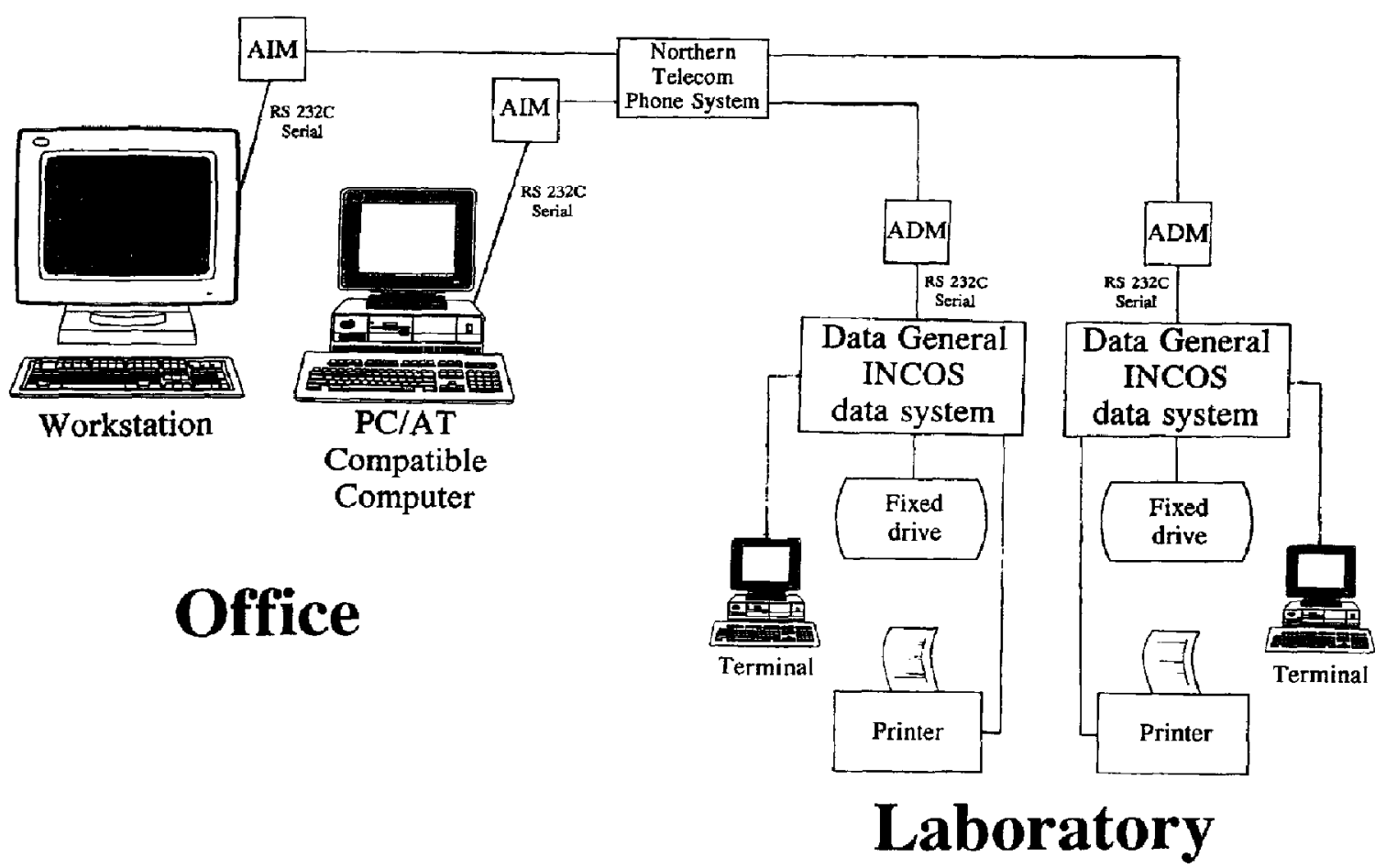

Figure 4. Schematic illustrating the connection of the Data General Nova 4X-based INCOS data systems to remote PCs and workstations via a Northern Telecom phone system.

on the network. Opening a connection between the ICIS data system and a remote PC or UNIX work station on the network can be readily accomplished using the "telnet," "rlogin," or "rsh" commands. The "rlogin" and "rsh" commands allow automated $\log$-in for users on trusted hosts ${ }^{2}$ listed in the "/etc/hosts.equiv" file on the DECstation 2100.

For access to the ICIS data system from a PC, the $X$-server software HCL eXceed Plus was used to allow the PCs to function as an X-Windows server. When HCL exceed Plus is run in $1024 \times 768 \times 256$ color mode, the PCs can operate in a manner that is essentially identical to the console of the DECstation 2100. In this particular application, automated $\log$-in from the PCs was implemented so that entry requires only a single command from the DOS prompt, and exit requires only a single keystroke. The simplest means for setting up remote terminal access using automated $\log$-in techniques to the ICIS data system is to use separate user log-ins for each remote access location. With separate log-ins, automatically starting the ICIS software can be accomplished with a simple script that sets the display and printer addresses, starts the Motif window manager (if desired), resets the ICIS intertask communications daemon, and starts ICIS Executive.

\footnotetext{
${ }^{2}$ See the Ultrix manual pages for " $r$ sh" and "rlogin."
}

Using this method allows remote terminal access from PCs that is identical to that available at the main console of the DECstation 2100 or with any desired degree of customized access for that particular terminal. Note that these techniques work equally as well with the PC-based data system for the Magnum ion trap detector.

Remote access to the ICIS data system from a UNIX workstation is carried out in an identical manner to that used for PCs. The only differences are that UNIX workstations are likely to have an $X$-server and a window manager already running and therefore would not start these programs at the beginning of an ICIS session. Although starting an ICIS session on a UNIX workstation can be easily automated using the "rsh" or "rlogin" commands, exiting typically requires closing each ICIS window or icon separately because it is usually not desirable to exit the $\mathrm{X}$-server and window manager at the end of an ICIS session as is done with the PCs. Nevertheless, exiting an ICIS session on a remote UNDX workstation is still a simple task; two mouse clicks are all that is required to close each window or icon.

Remote printing on a network printer is very easy from an ICIS session. Once the address of the printer has been made available to the DECstation 2100 in the file "/etc/printcap," the printer can be selected in the "Print..." menu of the ICIS Executive window. Note that the ICIS software calls the numbered aliases, 


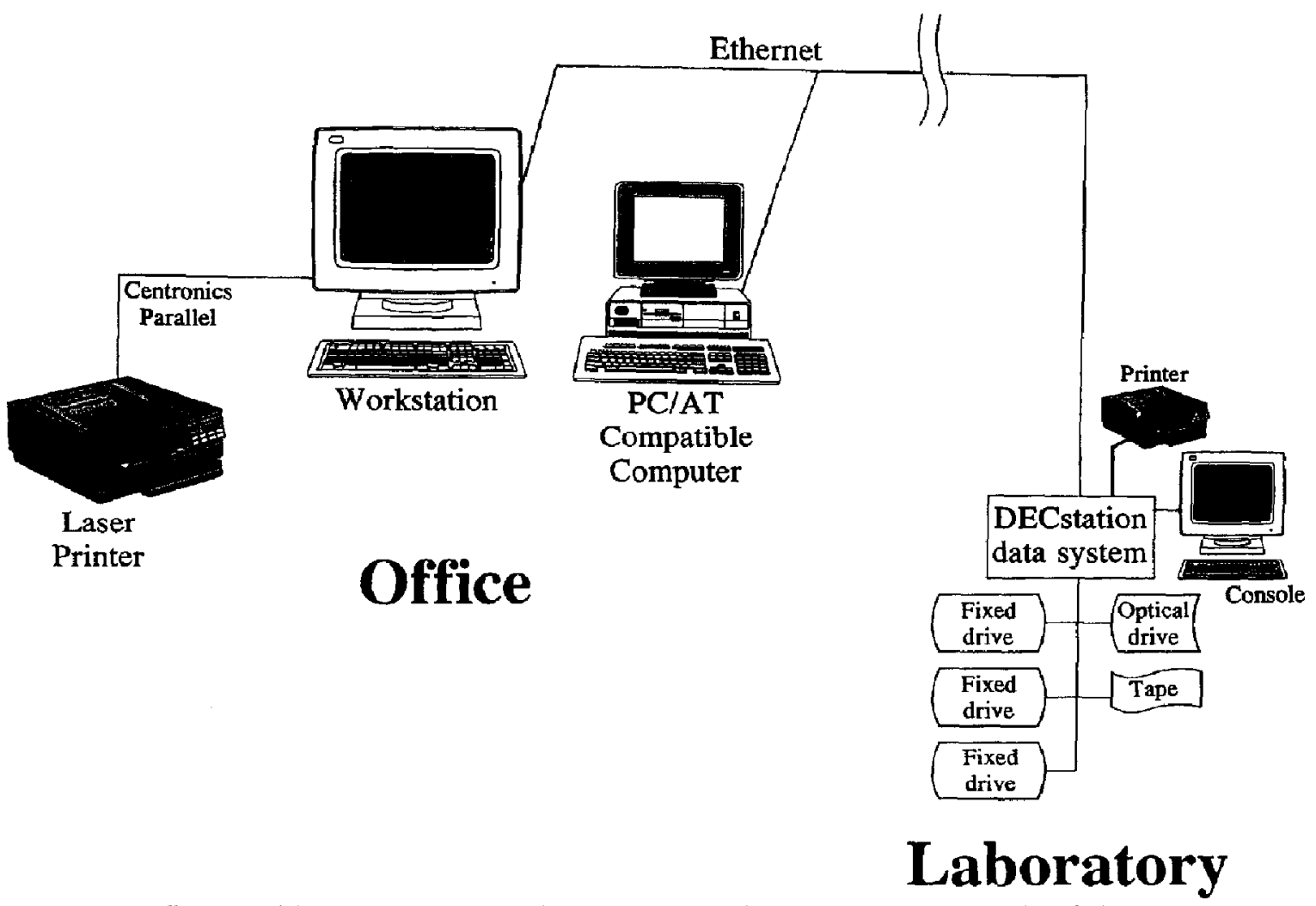

Figure 5. Schematic showing the Ethernet connection of the DECstation 2100-based ICIS data system to remote PCs, workstations, and printers. This link makes all of the capabilities of the data system available at remote locations.

and therefore care must be taken to specify these in the definition given in "/etc/printcap."

Use of these connectivity techniques for remote terminal access and remote printing allows the full use of all DEC and ICIS applications at remote terminals while permitting all other simultaneous uses of the DECstation 2100, including data acquisition. The DECstation as supplied by Firnigan-MAT utilizes a twouser version of the Ultrix operating system, which limits the simultaneous use of the ICIS data system to one main console user and two remote users. In all but the most demanding of circumstances, being limited to three simultaneous users of one data system should not be a problem.

An alternative for remote processing ion trap or INCOS data is to run a third-party software product, such as Benchtop PBM, MassSpec, or other similar PC programs on office PCs and use PC-NFS to mount the data storage drive, "/usr/users / netdrive," on the DECstation 2100. Similarly, the program MassLib can be utilized on VAX systems or workstations to process data at remote locations. Use of this technique provides another means of access in addition to those previously described; however, there can be some limitations in using these software products. Often, not all data file formats can be processed with this software. Also, network printing may be available but only via limited printer port definitions and to a very limited selection of printers.

\section{Conclusions}

The application of computer connectivity techniques to mass spectrometer data systems facilitated the achievement of the following two important objectives: (1) to electronically archive mass spectrometry data at a central location on a high-capacity, fast-access device that allows rapid retrieval of archived data from any computer on the network, and (2) to allow access to mass spectrometry data and data processing and printing functions from remote locations without affecting simultaneous data acquisition at the instruments. It is somewhat difficult to quantify the improvements gained by networking our mass spectrometer data systems in light of considerable improvements in efficiency made recently by automating sample intro- 
duction and data acquisition [10]. Nevertheless, by successfully completing these links, we noted a substantial improvement in productivity through greatly increased access to all required information when and where it is needed. Furthermore, the mass spectrometer data systems are readied for their eventual incorporation into a LIMS. Our most immediate plans for the development of a LIMS involve the installation of a dedicated server for processing mass spectrometry data and an optical disk jukebox for permanent archival of all analytical data.

Although the techniques applied here have worked well, there is still considerable work left to be done in the area of standardization. Completion of the work presented here depended heavily on the standardization of communication hardware. Before similar work can be successfully implemented in a multivendor environment, agreement must be reached on the standardization of a data transfer format. Recent work on the netCDF transfer mechanism for mass spectrometry data suggests that good progress is being made in the area of data formats [5,11]. Similarly, the widespread successful use of LIMS, where multiple vendors and analysis types are integrated, depends on some standardization of both data formats and computer platforms. Continued persistence in this area should also result in substantial standardization progress and, eventually, highly useful systems.

\section{Acknowledgments}

The authors acknowledge Ray Christopher of Finnigan-MAT for advice on the use of TFTP with SuperINCOS and the beta version 2 TFTP software. Dale $H$. Chidester is acknowledged for introducing the HCL eXceed $X$-server software for PCs at American Cyanamid. Richard $S$. Wayne is acknowledged for providing the time of his group members (P.V.R. and D.H.C.) for work on this project.

\section{References}

1. Szecsei, D. Am. Lab. 1991, 23, 30.

2. Williams, D.; Porter, C. Int. Lab. 1989, 19, 46.

3. Millard, B. J. Spectroscopy 1988, 3, 51.

4. Johnson, R. L.; Taylor, L. C. E. Procedings of the 39th ASMS Conference on Mass Spectrometry and Allied Topics; Nashville, TN, May 19-24, 1991; p 1227.

5. Stranz, D.; Campbell, S.; Cluristopher, R.; Watt, J.; Zackett, D. Pittsburgh Conference; New Orleans, LA, 1992; paper 037.

6. Lysakowski, R. Pittsburgh Conference; New Orleans, LA, 1992; paper 039.

7. Raffaelli, A.; Bruins, A. P. Rapid Commun. Mass Spectrom. 1992, 6, 582.

8. Mellerio, G. G.; Vago, G. M. Org. Mass Spectrom. 1991, 26, 985.

9. Stevens, R. W. UNIX Network Programming; Prentice Hall: Englewood Cliffs, NJ, 1990.

10. Hayward, M. J.; Snodgrass, J. T.; Thomson, M. L. Rapid Commun. Mass. Spectrom. 1993, 7, 85.

11. Hayward, M. J. Rapid Commun. Mass Spectrom. 1992, 6, 545. 